\title{
Take a deep breath but don't relax: Anesthesia for thymectomy in myasthenia gravis
}

\author{
Robert J. Korst, MD, MBA \\ From the Department of Thoracic Surgery, Mount Sinai Health System, Icahn School of Medicine at Mount Sinai, \\ New York, NY; Valley/Mount Sinai Comprehensive Cancer Care, Paramus, NJ; and Department of Surgery, \\ The Valley Hospital, Ridgewood, NJ. \\ Disclosures: Author has nothing to disclose with regard to commercial support. \\ Received for publication Dec 5, 2017; accepted for publication Dec 18, 2017; available ahead of print Feb 2, 2018. \\ Address for reprints: Robert J. Korst, MD, MBA, 1 Valley Health Plaza, Paramus, NJ 07652 (E-mail: korsro@ \\ valleyhealth.com). \\ J Thorac Cardiovasc Surg 2018;155:1890 \\ $0022-5223 / \$ 36.00$ \\ Copyright $(2) 2017$ by The American Association for Thoracic Surgery \\ https://doi.org/10.1016/j.jtcvs.2017.12.083
}

The anesthetic management of patients undergoing thymectomy for myasthenia gravis $(\mathrm{MG})$ remains problematic due to the risk of precipitating myasthenic crisis (MC) postoperatively with prolonged need for mechanical ventilation. Patients with MG are often hypersensitive to the use of neuromuscular blocking agents that are administered to facilitate the delivery of general, endotracheal anesthesia, oping MC after thymectomy has been reported to be in the range of approximately $5 \%$ to $20 \%{ }^{1-3}$

In this issue of the Journal, Jiang and colleagues ${ }^{4}$ present a retrospective, comparative study of 2 cohorts of patients that underwent thoracoscopic thymectomy for MG: one cohort received standard, general endotracheal anesthesia during the surgical procedure, whereas the other was intubated with a laryngeal mask airway and allowed to spontaneously ventilate. The authors found a significantly lower rate of postoperative $\mathrm{MC}$ and a shorter hospital stay in the mended on their continued work pioneering the performance of thoracic surgery in spontaneously breathing patients.

Although the study clearly demonstrates the feasibility of the spontaneously breathing approach in thymectomy for MG, drawing definitive conclusions from the comparison of the 2 groups regarding the advantages of spontaneous ventilation over conventional general endotracheal anesthesia is more challenging. Although the authors used propensity matching to try to eliminate selection bias in some not completely eliminate the effect of confounding factors in a retrospective series of patients. In addition, the 2 groups were managed differently in more ways other than the use of endotracheal intubation, especially regarding the uses of neuromuscular blocking agents and postoperative epidural pain control. Furthermore, it is difficult in a retrospective study to accurately analyze successful weaning from and their effects can be unpredictable. The risk of develspontaneously breathing patients and should be comof the general baseline characteristics, this approach will

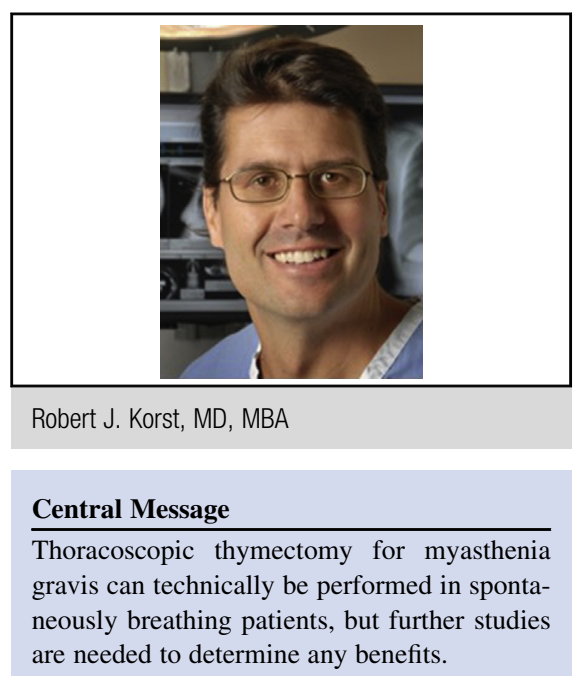

See Article page 1882. mechanical ventilation in the absence of a prospective protocol. Finally, the ability to draw conclusions regarding the length of hospital stay when factors other than medical necessity influence hospital discharge policies is extremely limited, which represents an issue in some countries outside of the United States.

Despite these concerns, strategies aimed at determining the best anesthetic management of patients with MG undergoing thymectomy warrant further investigation. Although the authors have demonstrated technical feasibility of their approach to this problem, they will need to continue their work in the form of a prospective clinical trial, ultimately in a randomized fashion with established protocols to demonstrate any beneficial effect for these patients.

\section{References}

1. Li K-K, Qian K, Feng Y-G, Guo W, Tan Q-Y, Deng B. Predictive factors of prolonged mechanical ventilation, overall survival, and quality of life in patients with post-thymectomy myasthenic crisis. World J Surg Oncol. 2017;15:150-6.

2. Xue L, Wang L, Dong J, Yuan Y, Fan H, Zhang Y, et al. Risk factors of myasthenic crisis after thymectomy for thymoma patients with myasthenia gravis. Eur J Cardiovasc Surg. 2017;52:692-7.

3. Leuzzi G, Meacci E, Cusumano G, Cesario A, Chiappetta M, Dall'armi V, et al. Thymectomy in myasthenia gravis: proposal for a predictive score of postoperative myasthenic crisis. Eur J Cardiothorac Surg. 2014;45:e76-88.

4. Jiang L, Depypere L, Rocco G, Chen JS, Liu J, Shao W, et al. Spontaneous ventilation thoracoscopic thymectomy without muscle relaxant for myasthenia gravis: comparison with "standard" thoracoscopic thymectomy. J Thorac Cardiovasc Surg. 2018;155:1882-9.e3. 